\title{
MAGNETOOPTICAL STUDIES OF BULK DILUTED MAGNETIC SEMICONDUCTORS
}

\author{
JAN GAJ \\ Institute of Experimental Physics, Warsaw University \\ Hoża 69, 00-681 Warszawa, Poland
}

Recent developments in physics of large gap II-VI Diluted Magnetic Semiconductors (DMS) studied by means of magnetooptical methods are reviewed. Applicability of the Heisenberg Hamiltonian to describe the ion-carrier interaction in new DMS materials is discussed. The paper includes anisotropy related phenomena, new type of bound magnetic polarons found in Van Vleck paramagnetic ( $\mathrm{Cd}, \mathrm{Fe}$ ) Se and discusses application of magnetic field to study kinetics of Raman cascades in DMS.

PACS numbers: 78.20. s

\section{Introduction}

Several important developments of the last years have brought a new breath to the physics of diluted magnetic semiconductors (DMS), a subject that has entered in the phase of maturity. The first obviously important event was successful growth of quantum wells and superstructures with either the well or the barrier (or both) made of DMS alloys. It opened a new perspective in studies of DMS, widening the range of magnetic ion (mostly $\mathrm{Mn}^{++}$) mole fraction values, enabling to study a transition from $3 \mathrm{D}$ to $2 \mathrm{D}$ magnetic phenomena and giving new possibilities of determination of band offsets. In the present paper we shall omit this important area and focus our attention on a few arbitrarily chosen recent developments in physics of bulk large gap II-VI DMS, practiced with help of magnetooptical methods. 
a

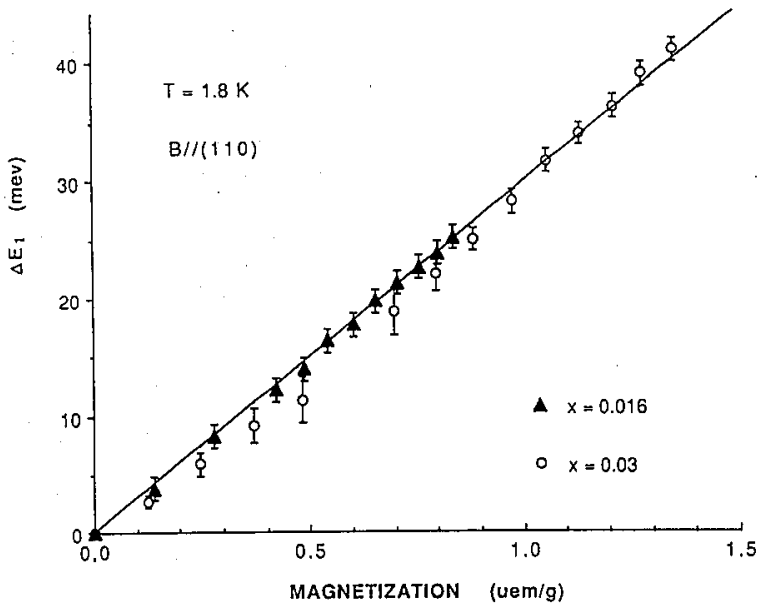

b

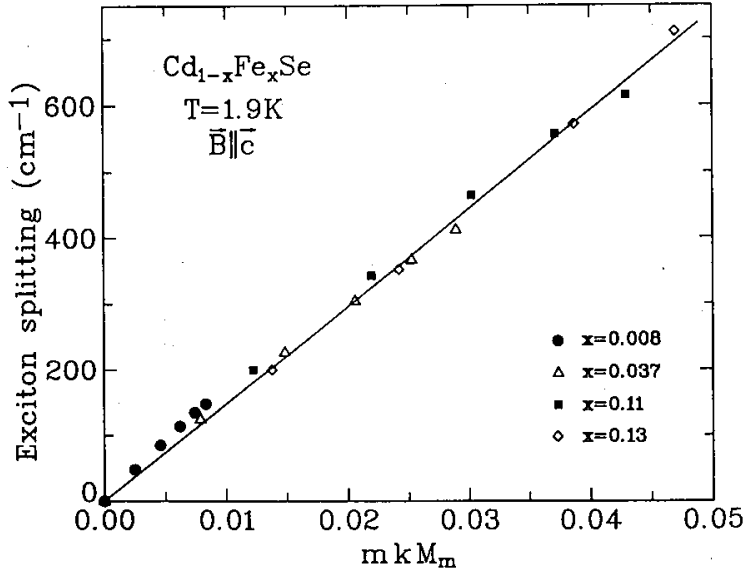

c

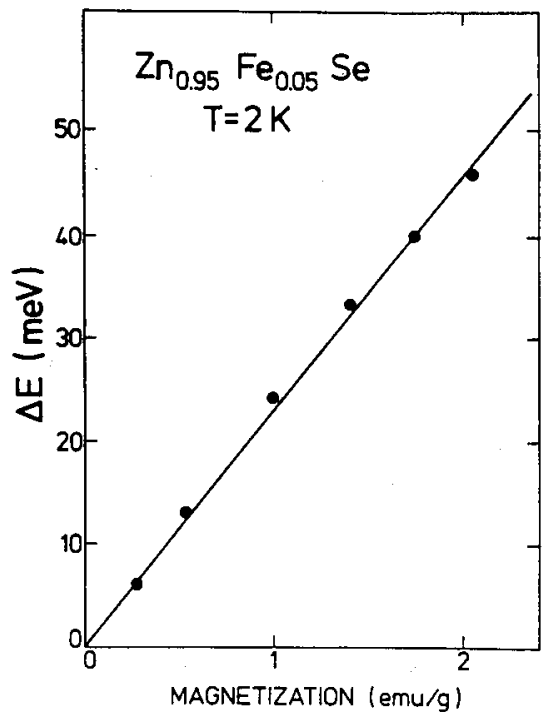




\section{Description of ion-carrier interaction}

This key interaction in DMS has usually been expressed in terms of a Heisenberg Hamiltonian

$$
H_{\mathrm{ex}}=-\sum_{\boldsymbol{i}} J\left(\boldsymbol{r}-\boldsymbol{R}_{i}\right) \boldsymbol{s} \cdot \boldsymbol{S}_{\boldsymbol{i}}
$$

where $r$ denotes position of the carrier, whereas $s$ and $S_{i}$ represent spin operators of the carrier and the ion, respectively, the latter occupying position $\boldsymbol{R}_{i}$ in the host semiconductor. Assuming this interaction form and several approximations natural for large-gap semiconductors one obtains in a mean field model a proportionality of Zeeman splittings of band states to the component along the magnetic field of the (thermally averaged) spin of the magnetic ion system [1]. This proportionality. has been checked first for (Cd, Mn) Te [2] by means of measurements of free exciton Zeeman splitting as a function of magnetization of the crystal has become a principal way of testing the applicability of Eq. (1) to new DMS materials. Several recent papers contain such tests performed on semiconductors with $\mathrm{Fe}^{++}$[3-5] and $\mathrm{Co}^{++}[6,7]$ ions. Their results, shown in Figs. 1a-1e show proportionality of Zeeman splittings to the magnetization of the samples, supporting the idea of Heisenberg description for the ion-carrier interaction. The problem has been recently analyzed theoretically by J. Blinowski and P. Kacman [8] who found that the form of the ion-carrier interaction depends crucially on symmetry of the lowest-lying group of the states of the ion. In case of $T_{2}$ symmetry the Heisenberg

d

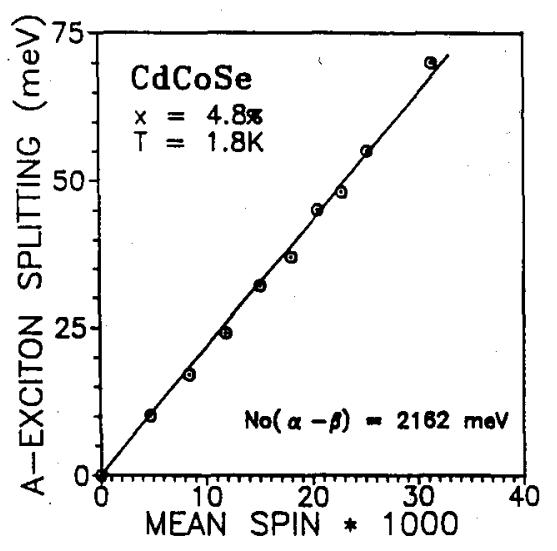

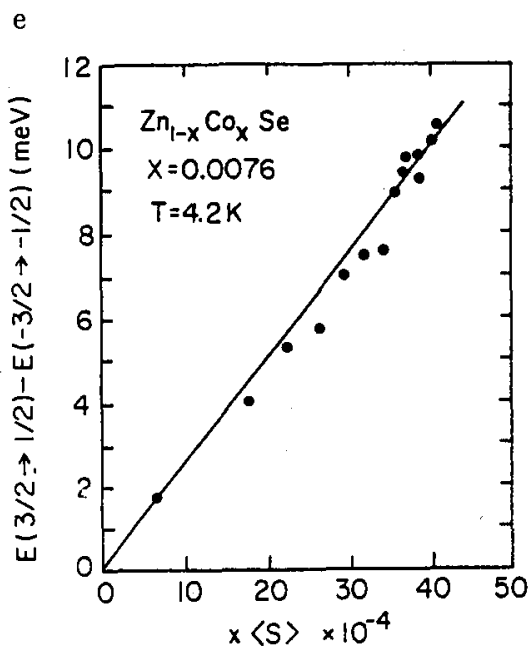

Fig. 1. Free exciton Zeeman splittings versus magnetization in various $\mathrm{DMS}$ with $\mathrm{Fe}^{\text {t+ }}$ and $\mathrm{Co}^{++}$ions: (a) (Cd, Fe) Te [5], (b) (Cd, Fe) Se [4], (c) (Zn, Fe) Se [3], (d) (Cd, Co) Se [7], (e) (Zn, Co) Se [6]. 
description (Eq. (1)) is inadequate. The form of the exchange interaction proposed in Ref. [8] is

$$
H_{\mathrm{ex}}=H_{1}(j \cdot S)+H_{2}(j, S, L),
$$

where $\boldsymbol{j}$ is the carrier momentum operator, $\boldsymbol{S}$ is the ion spin and $\boldsymbol{L}$ denotes a pseudo-momentum operator describing orbital movement in the $T_{2}$ term of the ion. Out of $\mathrm{Cr}^{++}, \mathrm{Mn}^{++}, \mathrm{Fe}^{++}$and $\mathrm{Co}^{++}$ions, the ground term has $T_{2}$ symmetry only for the $\mathrm{Cr}^{++}$ion. For the remaining ions Blinowski and Kacman find the Heisenberg approximation adequate, consistently with experimental results of Refs. [2-7]. The non-Heisenberg description for $\mathrm{Cr}^{++}$remains to be tested experimentally.

\section{Anisotropy related phenomena}

In contrast to the $\mathrm{Mn}^{++}$ions, which possess a half-filled $d^{5}$ shell, the remaining transition ions due to non-vanishing orbital moment exhibit a much larger sensitivity to their environment in the crystal. Therefore we can expect a number of anisotropy related effects in DMS containing those ions. Significant anisotropy of magnetization has been reported in $\mathrm{Fe}^{++}$based DMS: an axial anisotropy of about $30 \%$ in wurtzite $(\mathrm{Cd}, \mathrm{Fe}) \mathrm{Se}[9]$ and a cubic anisotropy of about $15 \%$ at $15 \mathrm{~T}$ in zincblende $(\mathrm{Cd}, \mathrm{Fe}) \mathrm{Te}[10]$. A particularly interesting example of such effects has been found by $\mathrm{A}$. Mycielski et al. [11] in quaternary $(\mathrm{Cd}, \mathrm{Fe})(\mathrm{Te}, \mathrm{Se})$, where depending on chemical composition of the four nearest anion neighbours of an iron ion, it may be exposed to a strong axial component of the crystal field. As a consequence, the Van Vleck paramagnetism exhibited by the $\mathrm{Fe}^{++}$ions both in CdSe and CdTe changes to the Curie paramagnetism observed e.g. in magnetic field dependence of the free exciton splitting and confirmed by disappearance of the characteristic $18 \mathrm{~cm}^{-1}$ FIR absorption line related to the $\mathrm{Fe}^{++}$states responsible for Van Vleck paramagnetism (Figs. 2, 3). One particular anisotropy related phenomenon - Jahn-Teller effect for the $\mathrm{Fe}^{++}$ion has once more attracted attention of researchers. In spite of its long history [12] the problem is still discussed: K. Lebecki et al. [13] find that its influence on the properties of a semiconductor with $\mathrm{Fe}^{++}$is very small, whereas A. Mycielski et al. [14] using FIR absorption data give precise values of Jahn-Teller (JT) energy and of the energy of the vibration involved $\left(2.5 \mathrm{~cm}^{-1}\right.$ and $28 \mathrm{~cm}^{-1}$ respectively). The small value of JT energy may be supported e.g. by the fact that magnetic field dependence of the $A_{1}-A_{2}$ and $A_{1}-E$ transition energies in a very similar system (Cd,Fe)Se [19] can be explained within a simple crystal field model, without JT effect. However, in this authors' view the obtained values still need to be confirmed.

\section{Van Vleck type bound magnetic polaron (BMP)}

Although the idea of bound magnetic polaron (a localized carrier coupled to magnetic ions within its orbit) is many years old [15], its first direct observation 


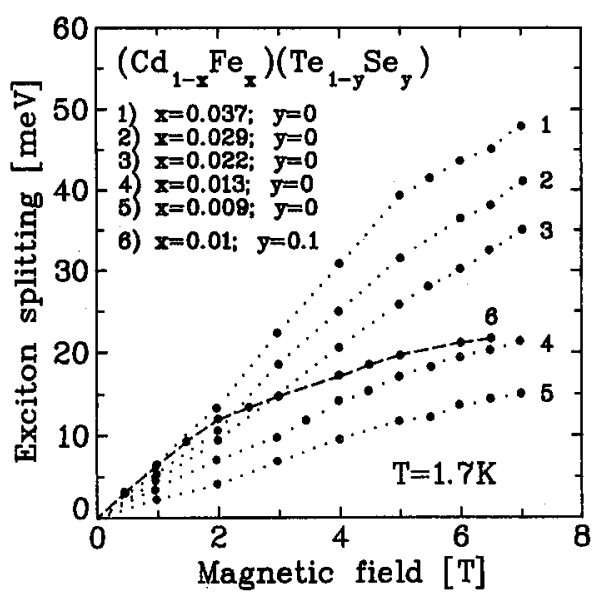

Fig. 2. Free exciton Zeeman splittings versus magnetic field for (Cd,Fe)Te (curves 1-5) and $(\mathrm{Cd}, \mathrm{Fe})(\mathrm{Te}, \mathrm{Se})$ (curve 6) [11].

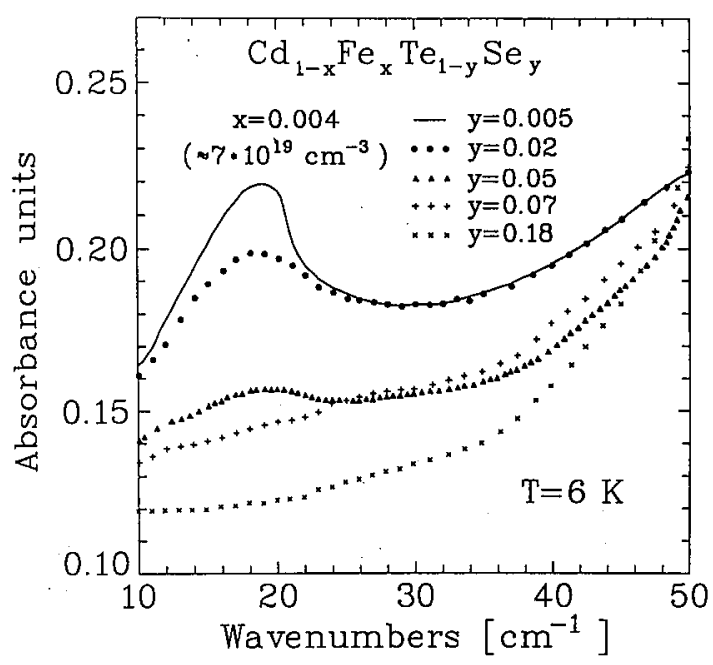

Fig. 3. FIR absorption spectra of $(\mathrm{Cd}, \mathrm{Fe})(\mathrm{Te}, \mathrm{Se})$ for various Se mole fractions [14].

has been done in DMS (Cd,Mn)Se by M. Nawrocki et al. [16] by means of spin flip Raman scattering (SFRS) on neutral donors: the spin flip energy measured as Stokes shift of the Raman line was different from zero without external magnetic field, demonstrating a local magnetization induced by the donor electron. The zero-field Stokes shift in SFRS has become the key test for existence of BMP in other materials with $\mathrm{Mn}^{++}$ions [15]. The first successful observation of SFRS on a $\mathrm{Fe}^{++}$containing DMS has been done on $(\mathrm{Cd}, \mathrm{Fe}) \mathrm{Se}$ by $\mathrm{D}$. Heiman et al. [9], who showed that the Stokes shift behaves to a good approximation linearly 
with macroscopic magnetization, disappearing in vanishing external magnetic field (Figure 4). D. Scalbert et al. [19] have shown that this result does not prove

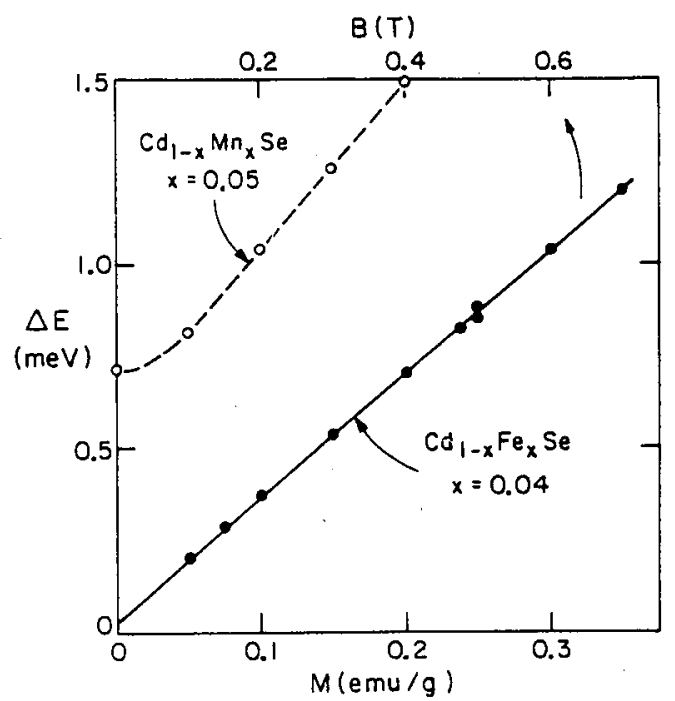

Fig. 4. Stokes shift in spin flip Raman scattering of (Cd,Fe)Se and (Cd,Mn)Se [9].

vanishing local magnetization since in Van Vleck paramagnets, to which (Cd, $\mathrm{Fe}$ )Se belongs, reversal of the carrier spin is not possible without reversing the local magnetization induced by the carrier. They have deduced the strength of the BMP effect from a non-crossing of two Raman lines: the SFRS one and that related to an internal excitation of the $\mathrm{Fe}^{++}$ion.

\section{Kinetics of Raman cascades}

Emission of optical phonons is known to be a very efficient energy relaxation channel. When creating excitons with kinetic energies many times larger than the optical phonon energy, cascade relaxation takes place [18]. D. Scalbert et al. [19] have reported such cascades where instead of phonons, internal excitations of $\mathrm{Fe}^{++}$ions are created. The cascades were observed directly in resonant Raman scattering (Fig. 5).

Two effects encountered when studying polarization of emitted light in a magnetic field: Hanle effect [20] (circular polarization) and resonant Faraday effect [21] (linear polarization) constitute valuable tools for determination of relaxation kinetics. Precession of a magnetic moment in the magnetic field serves as a clock in such experiments. Diluted magnetic semiconductors with their giant effective gyromagnetic factors open interesting perspectives in this respect since the clock in question runs very fast, enabling measurements of times of order of 1 ps. This feasibility, demonstrated in Ref. [19] for $\mathrm{Fe}^{++}$-related cascade relaxation, can hopefully be exploited in studies of other fast processes, e.g. phonon cascades. 


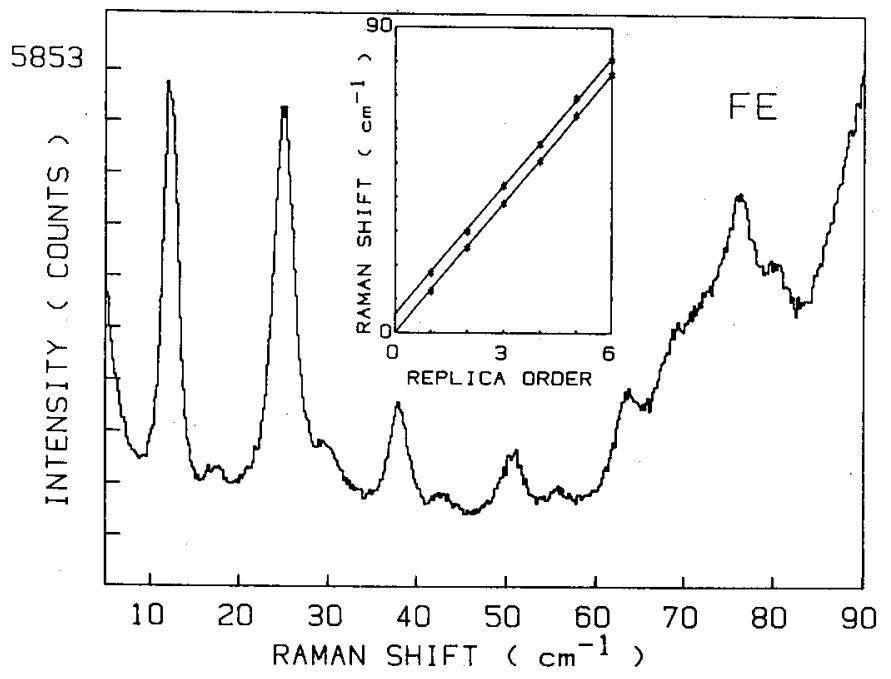

Fig. 5. Raman cascade involving intra-Fe ${ }^{++}$transitions [19].

\section{Conclusions}

The examples described above constitute only a small fraction of recent developments in the physics of diluted magnetic semiconductors. Very important areas have been omitted, such as 2D DMS structures, transport properties, carrier-induced bulk magnetic properties etc. Yet even the examples mentioned here alone evidence fertility of DMS physics both in the field of fundamental magnetic interactions and in finding new subtle effects.

\section{References}

[1] J.A. Gaj, J. Ginter, R.R. Galazka, Phys. Status Solidi B 89, 655 (1978).

[2] J.A. Gaj, R. Planel, G. Fishman, Solid State Commun. 29, 435 (1979).

[3] A. Twardowski, P. Glod, W.J.M. De Jonge, M. Demianiuk, Solid State Commun. 64, 63 (1987).

[4] A. Twardowski, K. Pakula, I. Perez, P. Wise, J.E. Cròw, Phys. Rev. B 42, 7567 (1990).

[5] C. Testelin, C. Rigaux, A. Mycielski, M. Menant, M. Guillot, to be published.

[6] X. Liu, B.T. Jonker, J.J. Krebs, G.A. Prinz, J. Warnock, J. Appl. Phys. 67, 4796 (1990).

[7] M. Nawrocki, F. Hamdani, J.P. Lascaray, Z. Golacki, J. Deportes, Solid State Comm. 77, 111 (1991).

[8] J. Blinowski, P. Kacman, in Proc. XX Int. Conf. Phys. Semicond., Thessaloniki 1990, Eds. E.M. Anastassakis, J.D. Joannopulos, World Scientific, Singapore 1990, Vol. 3, p. 1827. 
[9] D. Heiman, A. Petrou, S.H. Bloom, Y. Shapira, E.D. Isaacs, W. Giriat, Phys. Rev. Lett. 60, 1876 (1988).

[10] C. Testelin, A. Mauger, C. Rigaux, M. Guillot, A. Mycielski, Solid State Commun. 71, 923 (1989).

[11] A. Mycielski, W. Dobrowolski, A. Szadkowski, M. Borowiec, M. Arciszewska, C. Julien, C. Rigaux, M. Menant, in: Proc. XX Int. Conf. Phys. Semicond., Thessaloniki 1990, Eds. E.M. Anastassakis, J.D. Joannopulos, World Scientific, Singapore 1990, p. 702.

[12] G. Slack, S. Roberts, J.T. Vallin, Phys. Rev. 187, 511 (1969).

[13] K. Lebecki, A. Twardowski, to be published.

[14] A. Mycielski, M. Arciszewska, W. Dobrowolski, C. Rigaux, A. Mauger, C. Testelin, C. Julien, A. Lenard, M. Guillot, B. Witkowska, M. Menant, in Proc. XI Conf. Cond. Matter Div. EPS, Exeter 1991, to be published in Physica Scripta.

[15] P.W. Wolff, in Semiconductors and Semimetals, Eds. R.K. Willardson, A.C. Beer, Vol. Eds. J.K. Furdyna, J. Kossut, Academic Press, Boston 1988, Vol. 25, p. 413.

[16] M. Nawrocki, R. Planel, G. Fishman, R.R. Galazka, Phys. Rev. Lett. 46, 735 (1981).

[17] D. Scalbert, J.A. Gaj, A. Mauger, J. Cernogora, C. Benoit a la Guillaume, Phys. Rev. Lett. 62, 2865 (1989).

[18] see e.g. R.O. Callender, S.S. Sussman, M. Selders, R.K. Chang, Phys. Rev. $B$ 7, 3788 (1973).

[19] D. Scalbert, J.A. Gaj, A. Mauger, J. Cernogora, C. Benoit a la Guillaume, A. Mycielski, J. Cryst. Growth 101, 940 (1990).

[20] M.I. Dyakonov, V. Perel in Optical Orientation, Eds. F. Meier, B.P. Zakharchenya, North-Holland, Amsterdam 1984, p. 40.

[21] M.Nawrocki, R. Planel, C. Benoit a la Guillaume, Phys. Rev. Lett. 36, 1343 (1976). 